\title{
Inhibition of human cytomegalovirus major capsid protein expression and replication by ribonuclease $\mathrm{P}$-associated external guide sequences
}

\author{
QIUDI DENG, ${ }^{1,7}$ YUJUN LIU, ${ }^{2,3,7}$ XIN LI, ${ }^{1,7}$ BIN YAN, ${ }^{2,4,7}$ XU SUN ${ }^{5,6,7}$ WEI TANG, ${ }^{1,2}$ PHONG TRANG, ${ }^{2}$ \\ ZHU YANG, ${ }^{3,5,6}$ HAO GONG, ${ }^{5}$ YU WANG,${ }^{1,3,5}$ JIE LU, ${ }^{1}$ JUN CHEN, ${ }^{1}$ CHUAN XIA, ${ }^{1}$ XIWEN XING, ${ }^{1}$ \\ SANGWEI LU, ${ }^{2}$ and FENYONG LIU ${ }^{1,2}$ \\ ${ }^{1}$ Department of Biotechnology, College of Life Science and Technology, Jinan University, Guangzhou, Guangdong 510632, China \\ ${ }^{2}$ School of Public Health, University of California, Berkeley, California 94720, USA \\ ${ }^{3}$ Taizhou Institute of Virology, Taizhou, Jiangsu 225300, China \\ ${ }^{4}$ School of Pharmacy, Shandong University of Traditional Chinese Medicine, Jinan, Shandong 250355, China \\ ${ }^{5}$ Jiangsu Affynigen Biotechnolgies Inc, Taizhou, Jiangsu 225300, China \\ ${ }^{6}$ Guangzhou Qinheli Biotechnolgies Inc, Guangzhou, Guangdong 510600, China
}

\begin{abstract}
External guide sequences (EGSs) signify the short RNAs that induce ribonuclease $\mathrm{P}$ (RNase P), an enzyme responsible for processing the $5^{\prime}$ termini of tRNA, to specifically cleave a target mRNA by forming a precursor tRNA-like complex. Hence, the EGS technology may serve as a potential strategy for gene-targeting therapy. Our previous studies have revealed that engineered EGS variants induced RNase $P$ to efficiently hydrolyze target mRNAs. In the present research, an EGS variant was designed to be complementary to the mRNA coding for human cytomegalovirus (HCMV) major capsid protein (MCP), which is vital to form the viral capsid. In vitro, the EGS variant was about 80 -fold more efficient in inducing human RNase $P$ mediated cleavage of the target mRNA than a natural tRNA-derived EGS. Moreover, the expressed variant and natural tRNA-originated EGSs led to a decrease of MCP expression by $98 \%$ and $73 \%-74 \%$ and a decrease of viral growth by about 10,000- and 200-fold in cells infected with HCMV, respectively. These results reveal direct evidence that the engineered EGS variant has higher efficiency in blocking the expression of HCMV genes and viral growth than the natural tRNA-originated EGS. Therefore, our findings imply that the EGS variant can be a potent candidate agent for the treatment of infections caused by HCMV.
\end{abstract}

Keywords: external guide sequence; human cytomegalovirus; RNase P; gene-targeted therapy

\section{INTRODUCTION}

Human cytomegalovirus (HCMV), a human $\beta$-herpesvirus, causes congenital defects in neonates and severe diseases in immunosuppressed and immunocompromised hosts, particularly AIDS patients and transplantation recipients (Mocarski et al. 2013). Moreover, viral persistent/latent infection, which is commonly found in blood progenitor cells and leukocytes, plays an important role in HCMV pathogenesis and has been associated with various HCMV-associated disorders (Söderberg-Nauclér et al. 1997; Zhu et al. 2018). The absence of a vaccine for preventing HCMV infection and the emergence of resistant virus strains have

\footnotetext{
${ }^{7}$ These authors contributed equally to this work.

Corresponding authors: liu_fy@berkeley.edu, xingxiwen@jnu. edu.cn, sangwei@berkeley.edu

Article is online at http://www.rnajournal.org/cgi/doi/10.1261/rna. 069682.118.
}

demonstrated the necessity for new drug development and more effective therapeutic regimens (Drew 1992; Baldanti et al. 1996, 1998a,b). HCMV major capsid protein (MCP) plays a crucial role in HCMV capsid formation and generation of viral progeny (Gibson 1996; Dunn et al. 2003; Yu et al. 2003), and hence the therapy to block MCP expression may serve as a novel strategy for treatment of HCMV infection.

Nucleic acid-based gene interfering approaches are promising gene-targeting therapeutic methods against human diseases including viral infections (Stein and Cheng 1993; Gopalan et al. 2002; Scherer and Rossi 2003; Doudna

(C) 2019 Deng et al. This article is distributed exclusively by the RNA Society for the first 12 months after the full-issue publication date (see http://rnajournal.cshlp.org/site/misc/terms.xhtml). After 12 months, it is available under a Creative Commons License (Attribution-NonCommercial 4.0 International), as described at http:// creativecommons.org/licenses/by-nc/4.0/. 
and Charpentier 2014; Abudayyeh et al. 2017). For example, small interfering RNAs (siRNAs) were designed to hybridize to mRNA targets of several human viruses and induced the endogenous RNA-induced silencing complex (RISC) to degrade the target mRNAs, leading to knocking down gene expression and inhibiting viral infections (Jacque et al. 2002; Scherer and Rossi 2003; Wiebusch et al. 2004). Human RNase $P$ contains an RNA moiety (H1 RNA) and an auxiliary segment with at least ten proteins (Gopalan et al. 2002). RNase P-mediated degradation to remove the leader sequences from precursor tRNAs (pre-tRNAs) plays an essential role in the maturation of the $5^{\prime}$ end of a tRNA (Evans et al. 2006; Gopalan and Altman 2006; Marvin and Engelke 2009). RNase $P$ is capable of distinguishing the structure of substrates but not the sequences, and therefore this enzyme is able to degrade other substrates that contain a structure similar to a tRNA precursor structure. In principle, any two RNA molecules, when forming a complex that looks like a tRNA, are sufficient to allow for RNase P-mediated cleavage of one of the RNAs, the substrate RNA (Fig. 1A,B; Forster and Altman 1990; Yuan et al. 1992). The other RNA molecule refers to an external guide sequence (EGS), which must contain a sequence complementary to the substrate as well as other sequences/domains that interact with RNase P (Fig. 1; Liu 2010). Accordingly, any mRNA can be targeted by a custom-designed EGS RNA to direct RNase P for degradation. Previous studies have shown that EGSbased technology can serve as an approach to knock down gene expression in mammalian cells as well as bacteria (Yuan et al. 1992; Guerrier-

Takada et al. 1995; Plehn-Dujowich and Altman 1998; Ma et al. 2000; Hnatyszyn et al. 2001; Zhu et al. 2004). Our previous research indicated that human RNase P-mediated cleavage guided by EGSs could degrade the mRNA sequences of HCMV, herpes simplex virus 1 (HSV-1), Kaposi sarcoma-associated herpesvirus, and hepatitis B virus (HBV) in vitro and inhibited infection and gene expression of these viruses in cultured cells (Kawa et al. 1998; Zhou

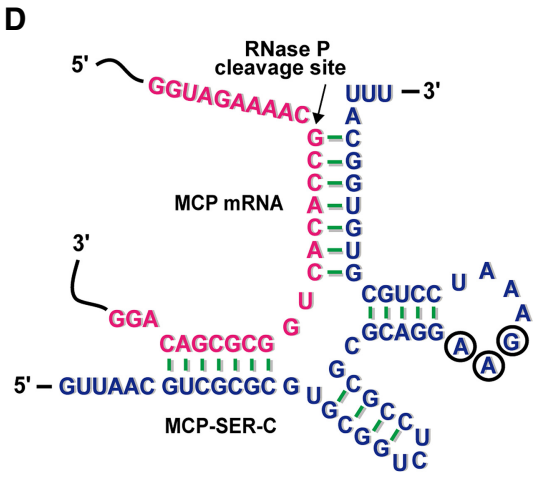

Target MCP mRNA : EGS MCP-SER-C

E

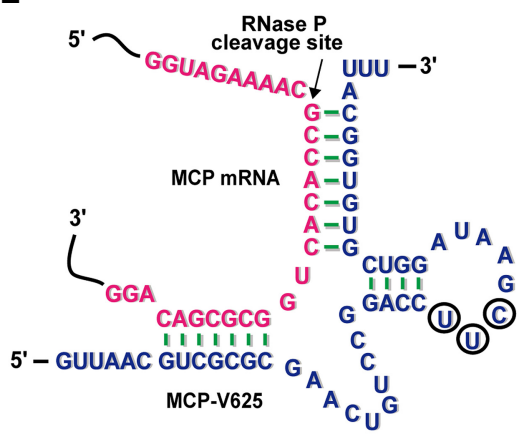

Target MCP mRNA : EGS MCP-V625

$\mathbf{F}$

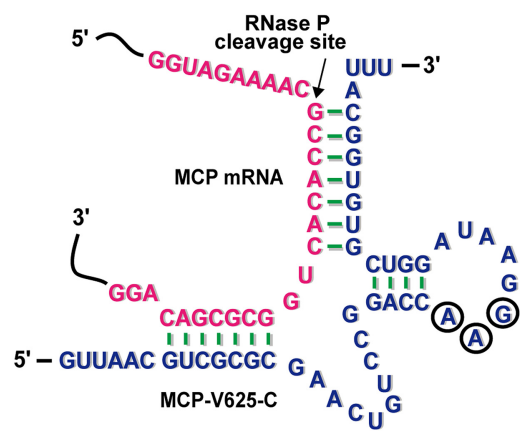

Target MCP mRNA : EGS MCP-V625-C

FIGURE 1. Schematic presentation of RNase $P$ substrates. (A) A natural substrate (pre-tRNA). (B) The binding of an EGS to a target RNA (e.g., mRNA) resembling a tRNA structure. (C-F) Hybridized complexes of the MCP mRNA sequence with MCP-SER, MCP-SER-C, MCPV625, and MCP-V625-C, respectively. The structures of MCP-SER and MCP-SER-C corresponding to the T-stem and-loop, and a variable domain of a tRNA originated from natural pre-tRNA ${ }^{\text {ser }}$, while MCP-V625 and MCP-V625-C were derived from EGS variant V625. The arrowhead marks the RNase P cleavage position. The blue color regions represent the EGS sequences.

et al. 2002; Zhu et al. 2004; Yang et al. 2006; Jiang et al. 2012; Xia et al. 2013).

For EGS-based technology to serve as a practical tool for knocking down gene expression as well as an agent for gene-targeting therapeutic strategies, it is crucial to enhance the efficacy of EGS-induced degradation by RNase $P$ both in vitro and in vivo. Novel EGS variants, generated through our previous in vitro selection procedure, 
were able to guide RNase $P$ to degrade the mRNA of HSV-1 thymidine kinase (TK) (Zhou et al. 2002). Furthermore, we showed that some of these variants induced RNase $P$ degradation of $m R N A$ s of $H C M V$ and hepatitis $B$ virus and inhibited infection of these viruses in cells (Yang et al. 2006; Jiang et al. 2012; Xia et al. 2013). While MCP is central for HCMV capsid formation and progeny production (Gibson 1996; Dunn et al. 2003; Yu et al. 2003), no studies of EGSs targeting HCMV MCP have been reported. It is not known whether EGS variants, when custom-designed for targeting the MCP mRNA, exert more effective suppression of the gene expression of HCMV and viral growth than a natural tRNA-originated EGS. The present study focused on investigating the efficacy of a custom-designed EGS inducing RNase $P$ to degrade the HCMV MCP mRNA and inhibit HCMV infection in cultured cells. Our results revealed that engineered EGS RNAs blocked HCMV MCP expression and viral growth more effectively than a natural tRNA sequence-derived EGS, resulting in a reduction of HCMV MCP expression up to $98 \%$ and the growth of HCMV as much as 10,000 times in HCMV-infected cells. Furthermore, our study provides direct evidence that engineered EGS variants targeting MCP mRNA may serve as a promising therapeutic strategy for suppression of HCMV infection.

\section{RESULTS}

\section{In vitro RNase P-mediated degradation of the HCMV MCP mRNA sequence directed by EGSs}

HCMV major capsid protein (MCP), which is essential for HCMV capsid formation and generation of viral progeny, is encoded by a viral UL86 open reading frame that is known to express a MCP mRNA transcript of $\sim 9 \mathrm{~kb}$ (Gibson 1996; Dunn et al. 2003; Yu et al. 2003). The intracellular mRNAs are mostly present in highly complex secondary structures and bind to proteins. Hence, selecting a targeted domain of the MCP mRNA sequence, which is approachable for interaction with EGSs for highly efficient degradation by RNase $P$, is vital. In previous studies (Yang et al. 2006; Jiang et al. 2012; Xia et al. 2013), we used dimethyl sulfate (DMS) to map the approachable domain of various mRNAs in cells (Liu and Altman 1995; Zaug and Cech 1995). In this study, we grew HCMV-infected human U251 cells and incubated them in DMS-containing culture media. It was expected that the nucleotides in the approachable regions of the targeted mRNA would be modified by DMS once it entered the cells. The total mRNA samples were extracted, and we then determined the approachable domains of the MCP mRNA subjected to DMS modification by primer extension experiments. With different primers bound to numerous viral and human mRNAs, we showed that all 33 mRNAs examined in the primer extension assays were modified by DMS in our experiments. Using primers specifically bound to the MCP mRNA regions, we showed that the MCP mRNA site that was 597 nucleotides downstream from the MCP translation start position was exceedingly susceptible to DMS modification (data not shown). Therefore, this site was selected as the RNase P cleavage position (Fig. 1C-F).

Previously, an in vitro selection procedure was performed to identify the EGS variants that could direct human RNase P-mediated degradation of the HSV-1 TK mRNA sequence with higher efficiency than a natural tRNA-originated EGS (Zhou et al. 2002). The ability of the screened-out EGS variants to target the HCMV MCP mRNA has not been studied. In this report, we characterized the activity of variant V625, one of the most efficient EGSs in guiding RNase $P$ degradation of the mRNA sequences of both the TK and MCP (see below, Table 1). EGS MCP-V625 was constructed by covalently joining the T-loop, a $\mathrm{T}$-stem, as well as a variable region of $\mathrm{V} 625$ with sequences partly complementary to the MCP mRNA sequence (Fig. 1E). EGS MCP-SER, based on the sequence of the natural tRNA ${ }^{\text {ser }}$, was designed in the same way as MCP-V625 (Fig. 1C). Two other EGSs, MCP-V625-C and MCP-SER-C, originated from MCP-V625 and MCP-SER, respectively,

TABLE 1. Kinetic analyses of RNase $P$ cleavage reactions for substrates ptRNA ${ }^{\text {ser }}$ or MCP mRNA sequence (mcp38) in the presence of different EGSs

\begin{tabular}{lllcc}
\hline Substrate & \multicolumn{1}{c}{$K_{\mathrm{m}}(\mu \mathrm{M})$} & $\begin{array}{c}V_{\max (\text { apparent) }} \\
\left(\mathrm{pmol} \cdot \mathrm{min}^{-1}\right)\end{array}$ & $\begin{array}{c}V_{\text {max(apparent }} / K_{\mathrm{m}(a p p a r a n t)} \\
\left(\mathrm{pmol} \cdot \mu \mathrm{M}^{-1} \cdot \mathrm{min}^{-1}\right)\end{array}$ & $2.7 \pm 0.4$ \\
\hline $\begin{array}{l}\text { ptRNA } \\
\text { Substrate mcp38 }\end{array}$ & $0.020 \pm 0.005$ & $0.054 \pm 0.016$ & & $K_{\mathrm{d}}(\mu \mathrm{M})$ \\
+ MCP-SER & $0.64 \pm 0.10$ & $0.020 \pm 0.008$ & $0.031 \pm 0.005$ & $1.8 \pm 0.4$ \\
+ MCP-SER-C & $\mathrm{ND}$ & $\mathrm{ND}$ & $<0.001$ & $1.8 \pm 0.4$ \\
+ MCP-V625 & $0.32 \pm 0.09$ & $0.80 \pm 0.20$ & $2.5 \pm 0.5$ & $0.019 \pm 0.004$ \\
+ MCP-V625-C & $\mathrm{ND}$ & $\mathrm{ND}$ & $<0.001$ & $0.019 \pm 0.004$ \\
\hline
\end{tabular}

The values are derived from the experiments. Each experiment, which was done in triplicate, was repeated three times. Experimental details are described in Materials and Methods. 
except that the T-loop containing the highly conserved region in these two control EGSs possessed three point mutations (5'-UUC-3' $\rightarrow$ AAG) (Fig. 1D,F). The natural tRNA sequences mostly contain these nucleotides (Jühling et al. 2009), which are believed to be essential for the interactions between human RNase $P$ and the tRNA domains (Gopalan and Altman 2006; Marvin and Engelke 2009). EGSs that contained these mutations failed to induce RNase P-mediated cleavage (Yuan and Altman 1994; Zhou et al. 2002; Zhu et al. 2004).

The DNA sequence templates coding for EGS RNAs were generated and then used to synthesize EGS RNAs by T7 RNA polymerase in vitro. The EGS RNAs were then mixed with both human RNase $P$ and the substrate, mcp38, which included the targeting sequence of MCP mRNA. Both MCP-SER and MCP-V625 showed human RNase P-mediated degradation of mcp38, respectively (Table 1). Further in vitro kinetic analysis experiments were performed to measure the catalytic activity and to obtain the $K_{m \text { (apparent) }}$ and $V_{\max (a p p a r e n t)}$ values and the overall cleavage efficiency $\left[V_{\max (a p p a r e n t)} / K_{m \text { (apparent) }}\right]$ of the EGSs in cleavage reactions. MCP-V625 had higher efficiency than MCP-SER by about 80-fold in inducing human RNase $P$ to cleave mcp38 (Table 1).

It is reasonable to suggest that the increased cleavage activities of human RNase $P$ probably resulted from additional tertiary binding between the EGS and mcp38 to further stabilize the complex structure of mcp38-EGS. To study whether the interaction of EGS variant MCP-V625 with the MCP mRNA sequence had higher affinity than that of the EGS (i.e., MCP-SER) originated from the sequence of a natural tRNA, gel-shift assays were performed to measure the constant of dissociation $\left(K_{d}\right)$. The results showed that the binding affinity of MCP-V625 to mcp38 was about 90 times higher than that of MCP-SER (Table 1). It is possible that the increased affinity and stability of the substrate-EGS complex may result from additional interactions between the EGS and mcp38, leading to better targeting activity of the EGS.

Human RNase P-mediated degradation of mcp38 with EGSs MCP-SER-C and MCP-V625-C was not apparent and was slower than that with MCP-V625 by at least $1 \times$ $10^{3}$-fold (Table 1). MCP-V625-C and MCP-SER-C shared identical antisense sequence (Fig. 1D,F) and similar in vitro binding affinities to mcp38 as MCP-V625 and MCP-SER, respectively (Table 1). Thus, MCP-V625-C and MCP-SER$C$ may serve as the control EGSs to account for the antisense effects.

\section{EGS expression in cultured cells}

To establish cell lines expressing EGS RNAs, the DNA sequences encoding MCP-SER, MCP-SER-C, MCP-V625, and MCP-V625-C were subcloned into the retroviral vector LXSN prior to transfection into PA317 (amphotropic pack- aging cells) to generate retroviral vectors. Retroviruses produced by these cells were then obtained and transfected into human U251 cells for construction of the cell lines stably expressing these EGSs. Another cell line expressing EGS TK112, which targeted TK mRNA (Kawa et al. 1998), was constructed. TK112 was chosen as a control EGS to determine whether an EGS RNA with an irrelevant guide sequence was capable of inducing human RNase $P$ to degrade the MCP mRNA sequence in cultured cells. In vitro experiments showed that TK112-mediated degradation of substrate mcp38 was not found (data not shown). In assays with MTT [3-(4,5-Dimethylthiazol-2-yl)-2,5-diphenyl tetrazolium bromide], the control cell line transfected with LXSN vector was similar to the EGS-expressing cell lines in viability and growth for at least $90 \mathrm{~d}$ (data not shown), indicating that all these EGSs had low cytotoxicity. Northern blot assays were carried out to detect the level of EGS RNAs in these generated cell lines, and H1 RNA, the human RNase P RNA subunit (Gopalan et al. 2002), was selected to be the internal control (Fig. 2). The cell lines exhibiting similar expression levels of EGS RNAs were chosen for further study.

\section{EGS-mediated suppression of HCMV MCP expression}

To measure the activities of EGS, HCMV was used to infect the cells in a multiplicity of infection (MOI) of 0.5-1, and then the total RNA samples, which were extracted from the culture cells, were studied by northern blot analysis for determination of the levels of MCP mRNA. HCMV 5kb-long immediate-early RNA (5 kb RNA), the expression of which is not regulated by MCP in the experimental conditions (Mocarski et al. 2013), served as the internal control for quantifying the MCP mRNA level (Fig. 3). A decrease of $98 \%$ and $76 \%$ (the mean of triplicate assays) in MCP mRNA expression was detected in the cells expressing MCP-V625 and MCP-SER, respectively (Table 2). In contrast, cells expressing MCP-SER-C, MCP-V625-C, or TK112 only

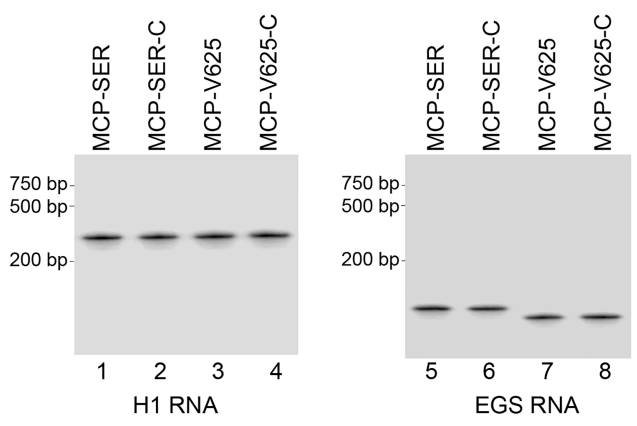

FIGURE 2. EGS RNA expression in culture cells. Northern blot assay was performed to analyze the RNA samples $(40 \mu \mathrm{g})$ from the subcloned cell lines that expressed MCP-SER (lanes 1,5), MCP-SER-C (lanes 2,6), MCP-V625 (lanes 3,7), and MCP-V625-C (lanes 4,8), respectively. 


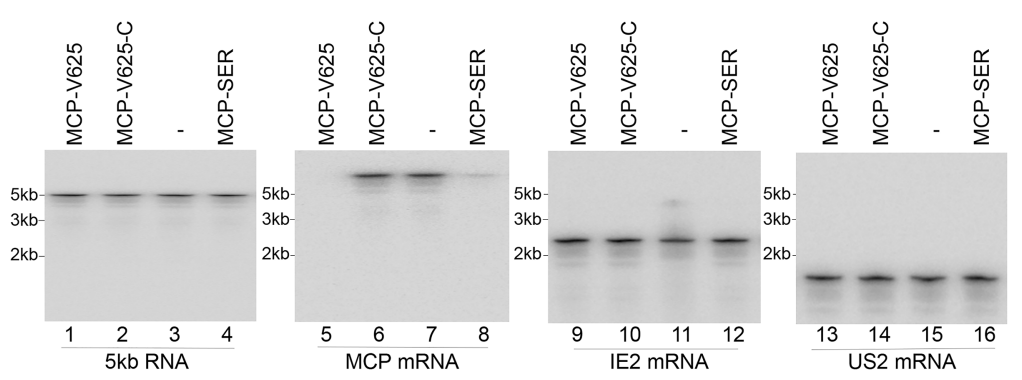

FIGURE 3. Northern blot analysis for the levels of HCMV mRNAs. Parental U251 cells (-, lanes $3,7,11,15$ ) and the cell lines expressing MCP-SER (lanes 4,8,12,16), MCP-V625-C (lanes $2,6,10,14$ ), and MCP-V625 (lanes 1,5,9,13) were infected with HCMV (Towne) at MOI of $0.5-1$. Total RNA samples were extracted from these cells. Northern blot analysis was performed to detect the expression of HCMV 5 kb transcript (5 kb RNA) (lanes 1-4), MCP mRNA (lanes 5-8), IE2 mRNA (lanes 9-12), and US2 mRNA (lanes 13-16).

exhibited a reduction of $<9 \%$. Thus, these observations suggested that inhibition of the targeted mRNA in the cells expressing MCP-SER and MCP-V625 was attributed to EGSs directing RNase P-mediated degradation. The antisense effect was probably the reason for the small decrease of MCP mRNA level in the cells expressing MCP-SER-C and MCP-V625-C, because these two control EGSs showed comparable interaction affinity as MCP-SER and MCPV625, respectively, with the targeted MCP mRNA but were not capable of directing RNase P for degradation.

We next determined the level of MCP protein expression in cells expressing EGSs using western blot assays. At 24-72 h post-infection, total protein samples were harvested from cells, separated in polyacrylamide gels, transferred to films, and then stained with antibodies against MCP (Fig. 4). For quantifying the level of MCP protein, human actin was used as an internal and loading control (Fig. 4, lanes 1-4). A decrease of $98 \%$ and $73 \%$ in the MCP protein expression was detected in cells expressing EGS MCP-V625 and MCP-SER, respectively (Table 2; Fig. 4). In comparison, $<9 \%$ decrease was observed in cells expressing control EGSs (Table 2; Fig. 4). The antisense effect was probably responsible for the low level of suppression in MCP protein expression in cells expressing MCP-SER-C or MCP-V625-C. Thus, the decrease in the levels of the MCP protein correlated well with the reduction of expression of $\mathrm{MCP}$ mRNA.

\section{Suppression of HCMV growth in cells expressing EGS by targeting MCP mRNA}

To evaluate the EGS-based suppression activity in viral replication, cells were infected with $\mathrm{HCMV}$ at an $\mathrm{MOI}$ of 1-5. Plaque assay experiments were carried out to measure the viral titers. On each day through $7 \mathrm{~d}$ post-infection, viral stocks from both the HCMV-infected cells and culture medium were harvested and used to infect human foreskin fibroblasts for determining the plaque forming units (PFU). On the fifth day post-infection, reduction of viral titers of at least 10,000- and 200-fold was detected in cells expressing EGS MCP-V625 and MCP-SER, respectively (Fig. 5). On the contrary, no noticeable decrease was observed in the cells expressing control EGSs MCPSER-C, MCP-V625-C, or TK112 (Table 2; Fig. 5). All these findings implied that the engineered EGS (i.e., MCPV625), through in vitro procedure selection, blocked HCMV growth with about 50 times more inhibition, compared with the EGS originated from a natural tRNA (i.e., MCP-SER).

\section{Suppression of HCMV capsid maturation by EGS- induced RNase P cleavage}

As the MCP plays a crucial role in HCMV capsid formation (Mocarski et al. 2013), reducing the MCP expression may

TABLE 2. Inhibition level of viral gene expression in EGS-expressing cells, as compared to U251 cells

\begin{tabular}{|c|c|c|c|c|c|c|c|}
\hline & \multirow[b]{2}{*}{ Viral gene class } & \multicolumn{6}{|c|}{ EGS RNA } \\
\hline & & U251 & TK112 & MCP-SER-C & MCP-V625-C & MCP-SER & MCP-V625 \\
\hline IE2 mRNA & $\alpha$ & $0 \%$ & $0 \%$ & $0 \%$ & $0 \%$ & $0 \%$ & $0 \%$ \\
\hline US2 mRNA & $\beta$ & $0 \%$ & $0 \%$ & $0 \%$ & $0 \%$ & $0 \%$ & $0 \%$ \\
\hline MCP mRNA & $\gamma$ & $0 \%$ & $0 \%$ & $5 \%$ & $8 \%$ & $76 \pm 9 \%$ & $98 \pm 9 \%$ \\
\hline UL80 & $\gamma$ & $0 \%$ & $0 \%$ & $0 \%$ & $0 \%$ & $0 \%$ & $0 \%$ \\
\hline $\mathrm{IE} 1$ protein & $\alpha$ & $0 \%$ & $0 \%$ & $0 \%$ & $0 \%$ & $0 \%$ & $0 \%$ \\
\hline UL44 protein & $\beta, \gamma$ & $0 \%$ & $0 \%$ & $0 \%$ & $0 \%$ & $0 \%$ & $0 \%$ \\
\hline MCP protein & $\gamma$ & $0 \%$ & $0 \%$ & $4 \%$ & $6 \%$ & $73 \pm 8 \%$ & $98 \pm 9 \%$ \\
\hline M99 protein & $\gamma$ & $0 \%$ & $0 \%$ & $0 \%$ & $0 \%$ & $0 \%$ & $0 \%$ \\
\hline
\end{tabular}

The values are obtained from the experiments. Each experiment, which was done in triplicate, was repeated three times. Only the standard deviations with values of more than $5 \%$ are shown. 

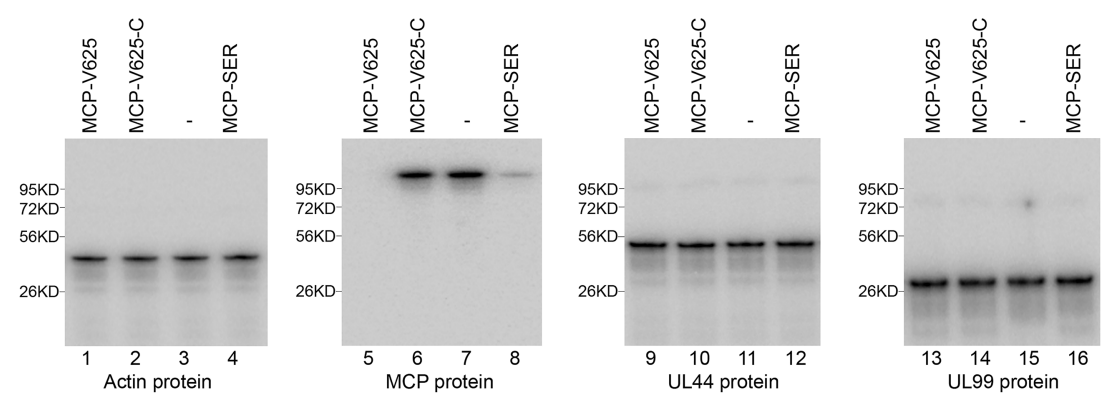

sequence was performed to determine the levels of intracellular viral DNA. The expression of human $\beta$-actin DNA was chosen to be the internal control. The HCMV genomic DNA is known to be episomal and does not integrate into the host genome, and therefore the amount of the intracellular viral DNA truly reflects the viral genome level (Mocarski et al. 2013). Moreover, we also determined the levels of encapsidated HCMV DNA to examine the degree of viral capsid maturation within the cells by subjecting the total DNA samples to digestion with DNase I. The unencapsidated DNA, which is not packaged in

lead to the inhibition of HCMV capsid maturation and therefore blockage of viral lytic replication. However, there is a possibility that the decrease of viral production in the EGS-expressing cells may not result from the EGS-induced RNase $\mathrm{P}$ degradation of the MCP mRNA but from other possible EGS-causing effects on HCMV lytic infection that are irrelevant to the outcome of EGS-induced suppression of MCP expression, which may include the suppression of viral immediate-early gene expression and genomic DNA replication. To resolve these matters and investigate the specific effect of EGS-induced RNase P degradation, two series of studies were performed to determine the relationships between the EGS-mediated degradation of the target MCP mRNA and the HCMV lytic infection step(s) that was blocked in the MCP-SER- and MCP-V625-expressing cells.

First, we evaluated whether the engineered EGSs affected the level of other viral gene expressions in HCMV-infected cells. Suppressing MCP expression was not expected to have an effect on the expression level of other HCMV genes, such as immediate-early $(\alpha)$, early $(\beta)$, and late $(\gamma)$ genes, which are not controlled by the MCP (Mocarski et al. 2013). Northern blot assays were performed to quantify the mRNA levels of HCMV IE2 (an $\alpha$ transcript), US2 (a $\beta$ transcript), and UL80 (a $\gamma$ transcript). Meanwhile, western blot assays were performed to examine the protein expression of viral IE1 (an $\alpha$ protein), UL44 [a late $(\beta \gamma)$ protein], and UL99 [a late $(\gamma)$ protein]. No notable disparity was detected in these gene expressions among the cells expressing different EGS RNAs (Table 2). Hence, MCP-SER and MCP-V625 expression seemed to specifically block MCP expression but not overall viral gene expression.

Second, we examined whether MCP-SER and MCPV625 RNAs had any effect on viral genomic replication and capsid formation. Total DNA was extracted from the cells infected with HCMV. A semiquantitative polymerase chain reaction (PCR) assay for the detection of HCMV IE1 the capsid, would be prone to digestion by DNase I, hence, the intracellular "encapsidated" viral DNA from the samples treated with DNase I were examined by PCR for determination of the level of HCMV IE1 DNA sequence.

No remarkable difference was observed in the levels of total intracellular HCMV DNA in the cells expressing EGSs (Fig. 6, lanes 1-4) when we used the total DNA samples without treatment of DNase I. These findings suggested that inhibition of the MCP expression by the EGSinduced RNase P cleavage did not affect the level of HCMV genomic DNA replication. On the contrary, when the samples were first incubated with DNase I and then assayed, the levels of "encapsidated" DNA were notably lower in the MCP-SER-expressing cells and much lower in the MCP-V625-expressing cells than those in cells expressing no EGS or control EGSs MCP-SER-C, MCP-V625-C, or TK112 (Fig. 6, lanes 5-8). These results implied that suppressing the MCP expression by MCP-SER and MCPV625 did not affect HCMV DNA replication but led to the

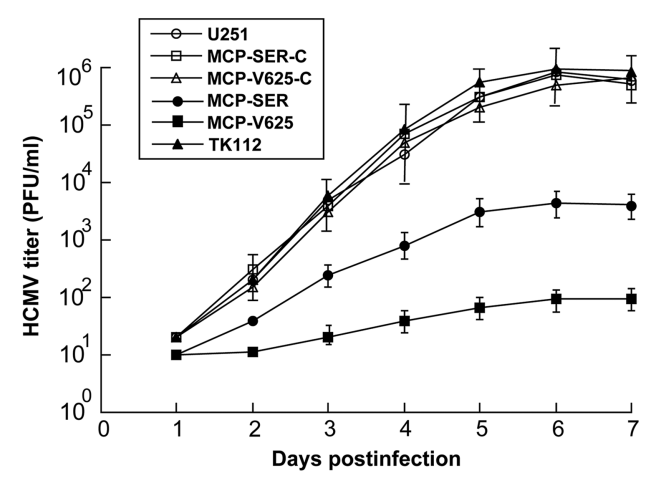

FIGURE 5. EGS-mediated inhibition of HCMV growth. Parental U251 cells and the cell lines expressing various EGSs were infected with $\mathrm{HCMV}$ at a $\mathrm{MOI}$ of 2, and virus stocks were obtained on each day through $7 \mathrm{~d}$ post-infection. The virus titer was determined by counting the plaque-forming unit (PFU) in HFFs. The values were expressed as the mean of three independent assays. 


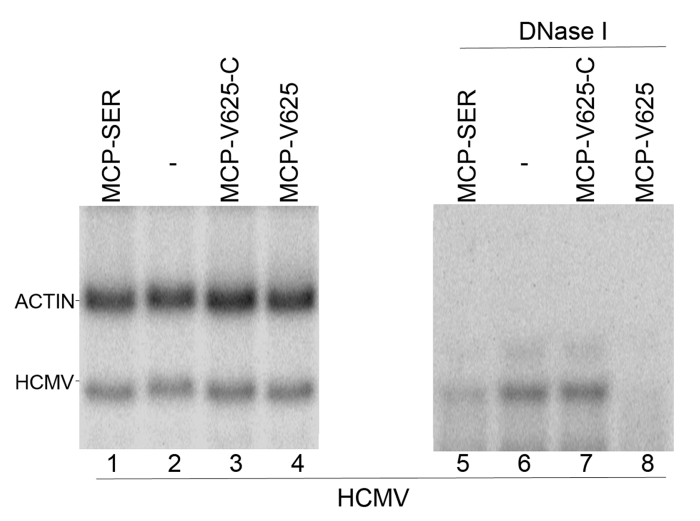

FIGURE 6. The levels of the intracellular total HCMV DNA $(A)$ and encapsidated DNA (B). Total DNA (lanes 1-4) or the DNA samples incubated with DNase I (lanes 5-8) were prepared from the HCMV-infected $(\mathrm{MOI}=1)$ cells either not expressing an EGS $(-$, lanes 2,6$)$ or expressing EGS MCP-SER (lanes 1,5), MCP-V625-C (lanes 3,7), or MCP-V625 (lanes 4,8). The HCMV IE1 sequence was amplified by $\mathrm{PCR}$, while the internal control was the human actin DNA sequence.

blockage of the HCMV capsid maturation/formation steps. Furthermore, the EGS variant (i.e., MCP-V625) created through the in vitro selection procedures had higher efficiency in inhibiting the HCMV capsid maturation/formation than a natural tRNA-originated EGS (i.e., MCP-SER).

\section{DISCUSSION}

The technology based on RNase-P-associated EGS represents a promising and attractive tool with high specificity and efficiency for gene interference. This strategy utilizes the intracellular RNase $P$, which is directed by engineered EGSs to degrade any target mRNAs (Gopalan and Altman 2006; Kim and Liu 2007). Moreover, EGS-directed RNase P-mediated cleavage has high specificity and lacks "irrelevant cleavage" that is usually associated with conventional antisense oligonucleotides inducing RNase $\mathrm{H}$-mediated cleavage (Ma et al. 2000). In order to be a practical therapy strategy, the EGSs must be efficient in interfering with gene expression. Generation of EGS variants with powerful efficacy may be necessary.

Limited available information is known about what restricts EGS-mediated targeting of an mRNA in cultured cells. Moreover, how to advance the effectiveness of EGSs is not completely understood. In our current study, EGS RNAs were custom-designed to target an approachable domain of the MCP mRNA. Furthermore, EGSs were expressed constitutively under the U6 RNA promoter with the intention for their expression in the nuclei. This design would enhance the chance for the EGS RNAs and their targeted mRNA sequence to bind to human RNase $\mathrm{P}$, which is known to be positioned in the nuclei (Gopalan and Altman 2006). In the described designs, we assumed that the activity of EGS-guided RNase P to degrade an mRNA is decided by its overall catalytic efficiency $\left(V_{\max } / K_{m}\right)$ in cultured cells. Enhancing the catalytic efficiency of EGS-induced cleavage by human RNase $P$ may result in stronger inhibition of the target mRNA expression in cultured cells.

Our findings demonstrated that a custom-designed EGS variant produced in an in vitro selection procedure (i.e., MCP-V625) had about 80 times higher efficiency [ $V_{\max }$ (apparent) $\left./ K_{\text {m(apparent) }}\right]$ in vitro in inducing RNase P cleavage of MCP mRNA sequence than the natural tRNA ${ }^{\text {ser }}$ sequence-derived EGS (i.e., MCP-SER). Further studies indicated that MCP-V625 was more effective $(98 \%$ vs. $73 \%-$ $76 \%$ ) in suppressing the expression of MCP in cultured cells, and about 50 times more efficient $(10,000$-fold vs. 200 -fold) in decreasing viral growth in cells infected with HCMV than MCP-SER. On the contrary, MCP-V625-C, MCP-SER-C, or TK112 showed far less efficacy $(<10 \%)$ in blocking the expression of MCP and reducing the growth of HCMV in cultured cells. In this study, MCP-V625-C and MCP-SER-C, which served as the control EGSs, showed relatively equivalent interaction affinity to substrate mcp38 as MCP-V625 and MCP-SER respectively, but exhibited little RNase $P$ guiding activity because of the T-loop mutations that prevented RNase $P$ interaction (Table 1; Fig. 1). These findings indicated that the suppression of HCMV gene expression and growth by MCP-V625 and MCP-SER was largely due to the specific action of these two EGSs in inducing RNase P-mediated cleavage but not the antisense effects of these EGSs or the other nonspecific effects resulted from the EGSs. Furthermore, the EGS variant (i.e., MCP-V625) showed higher efficiency $\left[V_{\text {max (apparent }} / K_{m \text { (apparent) }}\right]$ in guiding RNase $P$ to degrade the MCP mRNA sequence in vitro and was more effective in decreasing HCMV gene expression and growth in cultured human cells. This study provides supporting evidence to the hypothesis that enhancing the efficiency of EGS guiding human RNase $P$ to degrade a target mRNA in vitro may lead to enhancement of reducing gene expression in culture cells.

In order to develop highly efficient functional RNA molecules, a procedure by in vitro selection can be performed (Joyce 1992; Szostak 1992; Gold et al. 1995). In the present study, an EGS RNA molecular variant selected in vitro, which was screened out from a pool of EGS RNA molecules including sequences at random (Zhou et al. 2002), showed enhanced targeting efficacy in vitro and increased activity in suppressing HCMV MCP expression and viral growth in culture cells. Hence, the present research comes up with a new and important insight into developing highly efficient EGS RNAs by performing a selection procedure and designing the binding region of the EGS to a target mRNA.

Our previous studies have shown that engineered EGSs blocked viral infections in cultured cells (Kawa et al. 1998; Zhou et al. 2002; Zhu et al. 2004; Yang et al. 2006; Jiang et al. 2012; Xia et al. 2013). Compared to these previous 
studies, our current study is different in three ways. First, one of the most important findings in this report is EGS V625. Variant V625 has novel and unique sequences different from those at the T-loops and T-stems, and variable domains of natural tRNAs. Our study indicated that MCP-V625 had at least 90-fold more affinity for substrate mcp38 than MCP-SER (Table 1). Thus, EGS targeting efficacy may be enhanced by increasing the target mRNAEGS complex stability and formation, possibly achieved through creating additional tertiary interactions among different regions of the EGSs and the target mRNA.

Second, the EGS target in our study, MCP mRNA, is different from those in previous studies (Kawa et al. 1998; Zhou et al. 2002; Zhu et al. 2004; Yang et al. 2006; Jiang et al. 2012; Xia et al. 2013). HCMV MCP plays an essential role in viral replication and has conserved homologs among all viruses of the human herpesvirus family, which also includes HSV-1, Epstein Barr virus, and Kaposi sarcoma-associated herpesvirus (Mocarski et al. 2013). Our results implied that interfering with the expression of these proteins by the EGS strategy may serve as a promising approach against the infections of HCMV as well as other human herpesviruses. Studies using EGSs targeting HCMV MCP mRNA have not previously been reported. In this report, we showed a significant ( 10,000-fold) reduction of viral growth attributed to the EGS targeting activity, and provided direct evidence that suppression of HCMV MCP expression by RNase-P-associated EGSs may represent a promising antiviral therapeutic strategy.

Third, MCP-V625 appeared to be among the most active EGSs that have been constructed so far. In previous anti-HCMV studies, EGS variants, which targeted viral mRNAs other than MCP mRNA, had less than 40-fold more efficient targeting activity than the $\mathrm{RNA}^{\mathrm{SER}}$-originated EGSs in vitro, and inhibited the expression of viral gene targets by $98 \%$ and viral growth by not more than 7000 fold in cells (Yang et al. 2006; Jiang et al. 2012). In our current study, MCP-V625 was at least 80-fold more efficient in inducing RNase P-mediated degradation of MCP mRNA than MCP-SER in vitro, and inhibited MCP expression by $98 \%$ and viral growth by 10,000-fold in human cells (Tables 1, 2; Fig. 5). It has been shown that the efficacy of the EGS-based technology in cells can be substantially affected by the choice of the EGS targets and the mRNA sequence interacting with the EGS and RNase $P$ (Kawa et al. 1998; Zhou et al. 2002; Zhu et al. 2004; Yang et al. 2006; Jiang et al. 2012; Xia et al. 2013). Additional studies of EGSs targeting different sequences of various mRNAs should improve our understanding in the determining factors for the effectiveness of the EGS-based technology in cultured cells. These further studies will promote the development of EGS-based technology as potentially effective anti-HCMV therapeutic strategies.

Little is currently known about how the novel and unique sequences in V625 contribute to its increased targeting ac- tivity. EGS variant MCP-V625 binds to mcp39 about 90 times better and is about 80 times more active in inducing RNase P cleavage than MCP-SER, the EGS derived from a natural tRNA. It is possible that additional tertiary interactions may be present among the various unique sequences of MCP-V625 and mcp38, in order to enhance the binding of these sequences and the formation of mcp38-MCPV625 complex. The D-loop region of a tRNA (Fig. 1) has been shown to affect tRNA structure folding and RNase $P$ interaction, potentially by interacting with the other regions (e.g., T-loop and variant domains) of a tRNA (Evans et al. 2006; Gopalan and Altman 2006; Marvin and Engelke 2009). In the complex structure of mcp38-MCPV625, the 3' domain of mcp38 may serve as a structure resembling a part of the D-loop of a tRNA (Fig. 1E). It is conceivable that various regions of mcp38 and MCP-V625 may form additional interactions, resulting in enhancing the stability of the complex of mRNA-EGS and leading to a higher binding affinity and better EGS targeting efficacy. These potential additional interactions can be studied by generating and characterizing additional EGSs by introducing the structurally different variable loop and other MCP-V625 unique sequences into the natural tRNA-derived EGS (i.e., MCP-SER). Further investigation of these EGS variants should improve our understanding about the mechanism of how EGS RNAs induce RNase $P$ to efficiently degrade the target mRNA substrates.

\section{MATERIALS AND METHODS}

\section{Cells, viruses, and antibodies}

PA317 cells, U251 cells, and human primary foreskin fibroblasts (HFFs) were cultured in DMEM supplemented with 10\% (vol/vol) fetal bovine serum. As previously described (Trang et al. 2000; Dunn et al. 2003), HFFs and U251 cells were used to propagate HCMV (Towne strain). The human actin monoclonal antibody was acquired from Sigma Inc. The anti-UL44, IE1, MCP, and UL99 monoclonal antibodies of HCMV proteins were either obtained from Virusyn or kindly provided by Professor William Britt of the University of Alabama.

\section{To map the viral mRNA approachable domain in HCMV-infected cells}

To detect the approachable domain of viral mRNA, an in vivo mapping approach was carried out as previously described (Liu and Altman 1995; Zaug and Cech 1995). First, HCMV was chosen to infect U251 cells $(\mathrm{MOI}=1)$ for 8-24 h, and fresh DMEM media was used to wash cells prior to treatment of 1\% DMS for 5-10 min (Liu and Altman 1995; Zaug and Cech 1995). Cells were washed for three times using phosphate-buffered saline (PBS) supplemented with $1 \mathrm{mM} \beta$-mercaptoethanol, and then total RNA was obtained following the protocol of the RNA extraction kit (Qiangen China). To verify the DMS modification sites, primer extension assays were carried out following the procedures 
described previously (Trang et al. 2000; Yang et al. 2006; Jiang et al. 2012). The chemically synthesized oligonucleotide primers containing a 20 nt sequence complementary to several domains of the target mRNA were 5'-radiolabeled in the presence of $\gamma-\left[{ }^{32} \mathrm{P}\right]-\mathrm{ATP}$ by T4 polynucleotide kinase. A STORM840 Phosphorimager was used to analyze the blocked primer extension sites that represented the DMS-modified positions (Trang et al. 2000; Yang et al. 2006; Jiang et al. 2012).

\section{In vitro generation of EGS constructs for studies}

To construct the DNA sequence encoding the substrate mcp38, pGEM3zf (+) served as a template by PCR and AF25 (5'-GGA ATTCTAATACGACTCACTATAG-3') and m38 (5'-GGTTCAGT GCCTGTCGCGCCAGTGTGGCGTTTTCTACCCCTATAGTGAGT CGTATTA-3') as $5^{\prime}$ and $3^{\prime}$ primers, respectively. Moreover, PCR was used to synthesize the EGS-encoding DNA sequences with the previously constructed templates pV625 and pTK112 DNA (Kawa et al. 1998; Zhou et al. 2002). These EGS DNA sequences were under the control of the T7 RNA polymerase promoter (Kawa et al. 1998). For construction of PMCP-SER, the $5^{\prime}$ and 3' primer were oligoMCP-SER-5 (5'-GGAATTCTAATACGACTC ACTATAGGTTAAC GTCGCGCGTGCGGTCTCCGCGC-3') and oligoMCP-SER-3 (5'-AAGCTTTAAATGCCACACGCAGGATTTG AACCTGCGCGCGGAGACCGCAC-3'), respectively. For construction of PMCP-SER-C, the $5^{\prime}$ and $3^{\prime}$ primer were oligoMCPSER-5 and the oligoMCP-SER-C-3 (5'-AAGCTTTAAATGCCACA CGCAGGATTTCTTCCTGCGCGCGGAGACCGCAC-3'), respectively. For construction of pMCP-V625, the $5^{\prime}$ and $3^{\prime}$ primer were oligoMCP-V625-5 (5'-GGAATTCTAATACGACTCACTA TAGGTTAACGTCGCGCGAACTGTCCGGA-3') and oligoMCPV625-3 (5'-AAGCTTTAAATGCCACACGACCTATTCGAAGGTCC GGACAGTTC-3'), respectively. For construction of pMCP-V625$\mathrm{C}$, the $5^{\prime}$ and $3^{\prime}$ primer were oligoMCP-V625-5 and the oligoCSP-V625-C-3 (5'-AAGCTTTAAATGCCACACGACCTATTC CTTGGTCCGGACAGTTC-3'), respectively.

\section{In vitro studies of cleavage reactions and binding}

Human RNase P was extracted from HeLa cells following previously described protocols (Kawa et al. 1998; Zhou et al. 2002). The $\left[{ }^{32} \mathrm{P}\right]$-labeled mcp38 and the EGSs were incubated in buffer A (50 mM Tris, pH 7.4, $100 \mathrm{mM} \mathrm{NH}_{4} \mathrm{Cl}$, and $10 \mathrm{mM} \mathrm{MgCl}_{2}$ ) containing human RNase $\mathrm{P}$ at $37^{\circ} \mathrm{C}$. In order to examine the cleavage, the reaction samples were run in denaturing gels, and then analyzed with a STORM840 Phosphorimager. Moreover, assays were modified from the previously described protocols (Yuan and Altman 1994; Zhou et al. 2002) to examine the kinetic parameters under multiple turnover conditions. At regular intervals, aliquots of the reaction mixtures were withdrawn and polyacrylamide-urea gels were used to analyze the cleavage products. We determined the $K_{\mathrm{m} \text { (apparent) }}$ and $V_{\max \text { (apparent) values using }}$ the Lineweaver-Burk double-reciprocal plots (Liu and Altman 1994; Yuan and Altman 1994; Zhou et al. 2002).

To study the interaction of EGSs and the MCP mRNA sequence in vitro, a gel shift approach was performed following the previously described protocols (Pyle et al. 1990; Kawa et al. 1998; Zhou et al. 2002). Briefly, buffer B (50 mM Tris, pH 7.5, $100 \mathrm{mM}$ $\mathrm{NH}_{4} \mathrm{Cl}, 10 \mathrm{mM} \mathrm{MgCl}_{2}$, 3\% glycerol, $0.1 \%$ xylene cyanol, $0.1 \%$ bro- mophenol blue) was used to incubate various concentrations of EGSs for $10 \mathrm{~min}$, and an equal volume of different concentrations of substrate RNA was added. Binding of EGSs and mcp38 was examined by incubating the samples for 10-120 min, and then separating the samples in 5\% polyacrylamide gels using electrophoresis under 10 watts (Pyle et al. 1990). The equilibrium dissociation constant $\left(K_{\mathrm{d}}\right)$ values were calculated from a plot of the percentage of product bound versus the EGS concentration (Pyle et al. 1990; Kawa et al. 1998; Zhou et al. 2002), and the $K_{d}$ values were the average of the three independent assays.

\section{Construction of the EGSs-expressing cell lines}

The method of construction of the cell lines expressing EGSs was used following previously described protocols (Miller and Rosman 1989; Trang et al. 2000). PA317 (amphotropic cells) was transfected with the LXSN-MCP-SER, LXSN-MCP-SER-C, LXSNMCP-V625, LXSN-MCP-V625-C, and LXSN-TK112 retroviral vectors, respectively, and then these culture cell supernatants, which comprised retroviruses, were collected and subsequently used to infected U251 cells. At 48-72 h post-infection, cells were treated with neomycin $(500 \mu \mathrm{g} / \mathrm{mL})$ for $14 \mathrm{~d}$, and neomycin-resistant cells were chosen and cloned.

MTT assay was carried out to evaluate the cytotoxicity of the cell lines expressing EGSs. Parental U251 cells and the constructed cell lines were seeded in 96-well plates, and then appropriate volume of MTT solution [3-(4,5-Dimethylthiazol-2-yl)-2,5diphenyl tetrazolium bromide; Sigma; $5 \mathrm{mg} / \mathrm{mL}$ ] was added at different points in time. The absorbance was examined in the microplate reader at a wavelength of $570 \mathrm{~nm}$. Three independent experiments were carried out, with four parallel wells for each time. The morphology of the cells was also observed with a Nikon TE300 microscope.

Northern blot analysis experiments were performed to detect the EGS expression following the previously described protocols (Zhou et al. 2002; Bai et al. 2010). Total RNAs harvested from culture cells were separated in formaldehyde gels, transferred to nitrocellulose films, and then subjected to hybridization of $\left.{ }^{32} \mathrm{P}\right]-$ labeled DNA probes containing the MCP-V625 and MCP-SER sequences. A STORM840 Phosphorimager was used for analysis. The radiolabeled DNA probes detecting the expression of EGS RNAs were produced using a random primed radiolabeling kit according to the protocol of the manufacturer (Roche Applied Science) (Zhou et al. 2002; Bai et al. 2010).

\section{Assays of HCMV gene expression and viral growth in EGS-expressing cells}

To evaluate the EGS-mediated suppression of HCMV infection, viruses were used to infect cells ( $\mathrm{MOI}=1-5)$. We obtained viral stocks from the cells by collecting the cells and culture supernatants each day through $7 \mathrm{~d}$ post-infection, adding 10\% skim milk, and sonicating the mixture. Plaque assays were performed to determine the viral titers by infecting HFFs (Trang et al. 2000; Dunn et al. 2003). The viral titer results were the mean of three independent experiments.

To accurately determine the level of HCMV mRNA and proteins, northern and western blot analyses were carried out. As previously described (Zhou et al. 2002; Bai et al. 2010), both total viral 
proteins and mRNAs were harvested from the cells at 8-72 $\mathrm{h}$ postinfection. The radiolabeled DNA probes to detect the human $\mathrm{H} 1$ RNA, HCMV immediate-early $5 \mathrm{~kb}$ RNA transcript, IE2 mRNA, MCP mRNA, US2 mRNA, and UL80 mRNA were cloned from plasmids pH1 RNA, pCig27, plE2, pMCP, pCig38, and pUL80, respectively, and the detection of RNA expression was performed following previous methods (Zhou et al. 2002; Bai et al. 2010). In western blot assays, the total protein samples extracted from cells were run in polyacrylamide gels, transferred to nitrocellulose films, incubated with primary antibodies against HCMV proteins and human actin, followed by binding with HRP-conjugated secondary antibody, immunoreacted with the ECL substrates (GE Healthcare), and then analyzed with a STORM 840 Phosphorimager (Zhou et al. 2002; Bai et al. 2010).

\section{Determination of the level of intracellular viral genomic DNA}

Cells were mock-infected or infected with $\mathrm{HCMV}(\mathrm{MOI}=1)$, and then the viral inoculum was discarded after $2 \mathrm{~h}$ of incubation at $37^{\circ} \mathrm{C}$. At $48-96 \mathrm{~h}$ post-infection, the total DNA and encapsidated DNA (incubated with DNase I) were prepared as the templates for PCR following the protocols described previously (MatusickKumar et al. 1994). Human $\beta$-actin sequence was selected as the PCR amplified control with primers Actin5 (5'-TGACGGG GTCACCCACACTGTGCCCATCTA-3') and Actin3 (5'-CTAGAA GCATTGCGGTGGCAGATGGAGGG-3'), respectively. The level of HCMV DNA was examined by PCR amplifying the sequence of immediate-early IE1 with primers CMV3 (5'-CCAAGCGG CCTCTGATAACCAAGCC-3') and CMV4 (5'-CAGCACCATCCTC CTCTTCCTCTGG-3'), respectively. PCR reactions were carried out in the presence of radiolabeled $\alpha-\left[{ }^{32} \mathrm{P}\right]-\mathrm{dCTP}$. Moreover, the PCR products were run in polyacrylamide gels, and then analyzed with a STORM840 Phosphorimager. The PCR results were the mean of three independent assays.

\section{COMPETING INTEREST STATEMENT}

The authors declare that four authors, Z.Y., H.G., Y.W., and X.S., are employed by Jiangsu Affynigen Biotechnologies, Inc. and Guangzhou Qinheli Biotechnologies, Inc. This does not alter the authors' adherence to RNA policies. All other authors declare no competing financial interests.

\section{ACKNOWLEDGMENTS}

We are grateful to Marco Paliza-Corre, Giaphong Vu, Yong Bai, and Ting Wang for critical comments, reagents, and technical assistance. This research has been supported by grants from Guangdong Innovative and Entepreneurial Research Team Program (no. 2014ZT05S136), the National Mega Project on Major Infectious Disease Prevention (2012ZX10002006-003 and 2012ZX10004207), National Mega Project on Major Drug Development (2009ZX09103-678, 2012ZX09102301-004, 2012ZX0910330120, 2013ZX09102-031, 2014ZX09509001-001), National Natural Science Foundation of China (no. 31500137, no. 81672006, and no. 21708014), Antiviral Cooperative Innovation Center of Traditional Chinese Medicine at Shandong Province (XTCX2014B01-
09), Natural Science Foundation of Guangdong Province, China (no. 2015A030310515), Project for Construction of Guangzhou Key Laboratory of Virology (no. 201705030003), National Small Business Innovation and Research (SBIR) Program of China, the Technology R\&D Program of Jiangsu Province, China (BG2007035 and BG2008662), Open Research Fund Program of the State Key Laboratory of Virology of China (2015KF011 and 2018IOV006), a grant-in aid program for cultivation and innovation from Jinan University (no. 21616302), and the National Institutes of Health (RO1-DE023935 and RO1-DE025462).

Author contributions: Q.D., Y.L., X.L., B.Y., X.S., W.T., P.T., Z.Y., H.G., Y.W., J.L., J.C., C.X., X.X., S.L., and F.L. conceived and designed the experiments. Q.D., Y.L., X.L., B.Y., X.S., W.T., P.T., Z.Y., H.G., Y.W., J.L., J.C., and C.X. performed the experiments. Q.D., Y.L., X.L., B.Y., X.S., W.T., P.T., Z.Y., H.G., Y.W., J.L., J.C., C.X., X.X., S.L., and F.L. analyzed the data. Q.D., Y.L., X.L., B.Y., X.S., W.T., P.T., and Z.Y. contributed reagents/materials/analysis tools. Q.D., Y.L., X.L., B.Y., X.S., W.T., P.T., X.X., S.L., and F.L. wrote the paper. All authors reviewed the manuscript.

Received November 26, 2018; accepted February 21, 2019.

\section{REFERENCES}

Abudayyeh OO, Gootenberg JS, Essletzbichler P, Han S, Joung J, Belanto JJ, Verdine V, Cox DBT, Kellner MJ, Regev A, et al. 2017. RNA targeting with CRISPR-Cas13. Nature 550: 280-284. doi:10.1038/nature24049

Bai Y, Li H, Vu G, Gong H, Umamoto S, Zhou T, Lu S, Liu F. 2010. Salmonella-mediated delivery of RNase $P$ ribozymes for inhibition of viral gene expression and replication in human cells. Proc Natl Acad Sci 107: 7269-7274. doi:10.1073/pnas.0912813107

Baldanti F, Underwood MR, Stanat SC, Biron KK, Chou S, Sarasini A, Silini E, Gerna G. 1996. Single amino acid changes in the DNA polymerase confer foscarnet resistance and slow-growth phenotype, while mutations in the UL97-encoded phosphotransferase confer ganciclovir resistance in three double-resistant human cytomegalovirus strains recovered from patients with AIDS. J Virol 70: 13901395.

Baldanti F, Simoncini L, Talarico CL, Sarasini A, Biron KK, Gerna G. 1998a. Emergence of a ganciclovir-resistant human cytomegalovirus strain with a new UL97 mutation in an AIDS patient. AIDS 12: 816-818.

Baldanti F, Underwood MR, Talarico CL, Simoncini L, Sarasini A, Biron KK, Gerna G. 1998b. The Cys607->Tyr change in the UL97 phosphotransferase confers ganciclovir resistance to two human cytomegalovirus strains recovered from two immunocompromised patients. Antimicrob Agents Chemother 42: 444-446.

Doudna JA, Charpentier E. 2014. Genome editing. The new frontier of genome engineering with CRISPR-Cas9. Science 346: 1258096. doi:10.1126/science. 1258096

Drew WL. 1992. Cytomegalovirus infection in patients with AIDS. Clin Infect Dis 14: 608-615. doi:10.1093/clinids/14.2.608-a

Dunn W, Chou C, Li H, Hai R, Patterson D, Stolc V, Zhu H, Liu F. 2003. Functional profiling of human cytomegalovirus genome. Proc Natl Acad Sci 100: 14223-14228. doi:10.1073/pnas.2334032100

Evans D, Marquez SM, Pace NR. 2006. RNase P: interface of the RNA and protein worlds. Trends Biochem Sci 31: 333-341. doi:10 .1016/j.tibs.2006.04.007

Forster AC, Altman S. 1990. External guide sequences for an RNA enzyme. Science 249: 783-786. doi:10.1126/science.1697102

Gibson W. 1996. Structure and assembly of the virion. Intervirology 39: 389-400. doi:10.1159/000150509 
Gold L, Polisky B, Uhlenbeck O, Yarus M. 1995. Diversity of oligonucleotide functions. Annu Rev Biochem 64: 763-797. doi:10 .1146/annurev.bi.64.070195.003555

Gopalan V, Altman S. 2006. Ribonuclease P: structure and catalysis. In The RNA world, $3^{\text {rd }}$ ed. (ed. Gesteland R, et al.), Vol. 277, Chap. 6.1 (online only at http://rna.cshl.edu). Cold Spring Harbor Laboratory Press, Cold Spring Harbor, NY.

Gopalan V, Vioque A, Altman S. 2002. RNase P: variations and uses. J Biol Chem 277: 6759-6762. doi:10.1074/jbc.R100067200

Guerrier-Takada C, Li Y, Altman S. 1995. Artificial regulation of gene expression in Escherichia coli by RNase P. Proc Natl Acad Sci 92: 11115-11119. doi:10.1073/pnas.92.24.11115

Hnatyszyn H, Spruill G, Young A, Seivright R, Kraus G. 2001. Longterm RNase P-mediated inhibition of HIV-1 replication and pathogenesis. Gene Ther 8: 1863-1871. doi:10.1038/sj.gt.3301606

Jacque JM, Triques K, Stevenson M. 2002. Modulation of HIV-1 replication by RNA interference. Nature 418: 435-438. doi:10.1038/ nature00896

Jiang X, Chen YC, Gong H, Trang P, Lu S, Liu F. 2012. Ribonuclease Pmediated inhibition of human cytomegalovirus gene expression and replication induced by engineered external guide sequences. RNA Biol 9: 1186-1195. doi:10.4161/rna.21724

Joyce GF. 1992. Directed molecular evolution. Sci Am 267: 90-97. doi:10.1038/scientificamerican1292-90

Jühling F, Mörl M, Hartmann RK, Sprinzl M, Stadler PF, Pütz J. 2009. tRNAdb 2009: compilation of tRNA sequences and tRNA genes. Nucleic Acids Res 37: D159-D162. doi:10.1093/nar/gkn772

Kawa D, Wang J, Yuan Y, Liu F. 1998. Inhibition of viral gene expression by human ribonuclease P. RNA 4: 1397-1406. doi:10.1017/ S1355838298980918

Kim K, Liu F. 2007. Inhibition of gene expression in human cells using RNase P-derived ribozymes and external guide sequences. Biochim Biophys Acta 1769: 603-612. doi:10.1016/j.bbaexp .2007.09.001

Liu F. 2010. Ribonuclease P as a tool. In Ribonuclease P (ed. Liu F), pp. 257-276. Springer, New York.

Liu F, Altman S. 1994. Differential evolution of substrates for an RNA enzyme in the presence and absence of its protein cofactor. Cell 77: 1093-1100. doi:10.1016/0092-8674(94)90448-0

Liu F, Altman S. 1995. Inhibition of viral gene expression by the catalytic RNA subunit of RNase P from Escherichia coli. Genes Dev 9: 471-480. doi:10.1101/gad.9.4.471

Ma M, Benimetskaya L, Lebedeva I, Dignam J, Takle G, Stein CA. 2000. Intracellular mRNA cleavage induced through activation of RNase $P$ by nuclease-resistant external guide sequences. Nat Biotechnol 18: 58-61. doi:10.1038/71924

Marvin MC, Engelke DR. 2009. Broadening the mission of an RNA enzyme. J Cell Biochem 108: 1244-1251. doi:10.1002/jcb.22367

Matusick-Kumar L, Hurlburt W, Weinheimer SP, Newcomb WW, Brown JC, Gao M. 1994. Phenotype of the herpes simplex virus type 1 protease substrate ICP35 mutant virus. J Virol 68: 5384-5394.

Miller AD, Rosman GJ. 1989. Improved retroviral vectors for gene transfer and expression. Biotechniques 7: 980-982. 984-986, 989-990.

Mocarski ES, Shenk T, Griffiths PD, Pass RF. 2013. Cytomegaloviruses. In Fields virology (ed. Knipe DM, et al.), pp. 1960-2014. Lippincott-William \& Wilkins, Philadelphia.

Plehn-Dujowich D, Altman S. 1998. Effective inhibition of influenza virus production in cultured cells by external guide sequences and ribonuclease P. Proc Natl Acad Sci 95: 7327-7332. doi:10.1073/ pnas.95.13.7327

Pyle AM, McSwiggen JA, Cech TR. 1990. Direct measurement of oligonucleotide substrate binding to wild-type and mutant ribozymes from Tetrahymena. Proc Natl Acad Sci 87: 8187-8191. doi:10.1073/pnas.87.21.8187

Scherer LJ, Rossi JJ. 2003. Approaches for the sequence-specific knockdown of mRNA. Nat Biotechnol 21: 1457-1465. doi:10 $.1038 / \mathrm{nbt} 915$

Söderberg-Nauclér C, Fish KN, Nelson JA. 1997. Reactivation of latent human cytomegalovirus by allogeneic stimulation of blood cells from healthy donors. Cell 91: 119-126. doi:10.1016/S00928674(01)80014-3

Stein CA, Cheng YC. 1993. Antisense oligonucleotides as therapeutic agents—is the bullet really magical? Science 261: 1004-1012. doi:10.1126/science. 8351515

Szostak JW. 1992. In vitro genetics. Trends Biochem Sci 17: 89-93. doi:10.1016/0968-0004(92)90242-2

Trang P, Lee M, Nepomuceno E, Kim J, Zhu H, Liu F. 2000. Effective inhibition of human cytomegalovirus gene expression and replication by a ribozyme derived from the catalytic RNA subunit of RNase P from Escherichia coli. Proc Natl Acad Sci 97: 58125817. doi:10.1073/pnas.100101797

Wiebusch L, Truss M, Hagemeier C. 2004. Inhibition of human cytomegalovirus replication by small interfering RNAs. J Gen Virol 85: 179-184. doi:10.1099/vir.0.19453-0

Xia C, Chen YC, Gong H, Zeng W, Vu GP, Trang P, Lu S, Wu J, Liu F. 2013. Inhibition of hepatitis $B$ virus gene expression and replication by ribonuclease P. Mol Ther 21: 995-1003. doi:10.1038/mt .2013 .37

Yang YH, Li H, Zhou T, Kim K, Liu F. 2006. Engineered external guide sequences are highly effective in inducing RNase P for inhibition of gene expression and replication of human cytomegalovirus. Nucleic Acids Res 34: 575-583. doi:10.1093/nar/gkj431

Yu D, Silva MC, Shenk T. 2003. Functional map of human cytomegalovirus AD169 defined by global mutational analysis. Proc Natl Acad Sci 100: 12396-12401. doi:10.1073/pnas.1635160100

Yuan Y, Altman S. 1994. Selection of guide sequences that direct efficient cleavage of mRNA by human ribonuclease P. Science 263: 1269-1273. doi:10.1126/science.8122108

Yuan Y, Hwang ES, Altman S. 1992. Targeted cleavage of mRNA by human RNase P. Proc Natl Acad Sci 89: 8006-8010. doi:10 .1073/pnas.89.17.8006

Zaug AJ, Cech TR. 1995. Analysis of the structure of Tetrahymena nuclear RNAs in vivo: telomerase RNA, the self-splicing rRNA intron, and U2 snRNA. RNA 1: 363-374.

Zhou T, Kim J, Kilani AF, Kim K, Dunn W, Jo S, Nepomuceno E, Liu F. 2002. In vitro selection of external guide sequences for directing RNase P-mediated inhibition of viral gene expression. J Biol Chem 277: 30112-30120. doi:10.1074/jbc.M200183200

Zhu J, Trang P, Kim K, Zhou T, Deng H, Liu F. 2004. Effective inhibition of Rta expression and lytic replication of Kaposi's sarcoma-associated herpesvirus by human RNase P. Proc Natl Acad Sci 101: 9073-9078. doi:10.1073/pnas.0403164101

Zhu D, Pan C, Sheng J, Liang H, Bian Z, Liu Y, Trang P, Wu J, Liu F, Zhang CY, et al. 2018. Human cytomegalovirus reprogrammes haematopoietic progenitor cells into immunosuppressive monocytes to achieve latency. Nat Microbiol 3: 503-513. doi:10.1038/ s41564-018-0131-9 

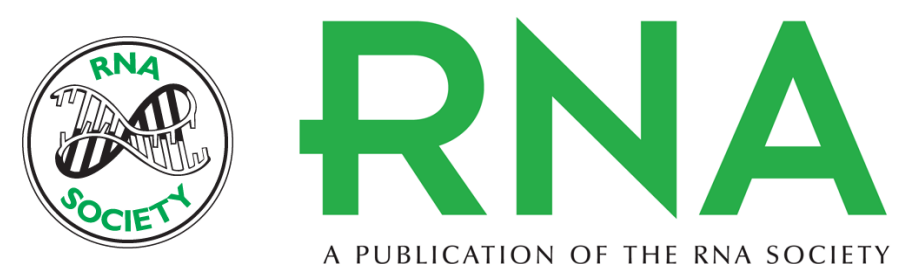

A PUBLICATION OF THE RNA SOCIETY

\section{Inhibition of human cytomegalovirus major capsid protein expression and replication by ribonuclease $\mathbf{P}$-associated external guide sequences}

Qiudi Deng, Yujun Liu, Xin Li, et al.

RNA 2019 25: 645-655 originally published online February 25, 2019

Access the most recent version at doi:10.1261/rna.069682.118

References This article cites 44 articles, 21 of which can be accessed free at: http://rnajournal.cshlp.org/content/25/5/645.full.html\#ref-list-1

Creative This article is distributed exclusively by the RNA Society for the first 12 months after the Commons full-issue publication date (see http://rnajournal.cshlp.org/site/misc/terms.xhtml). After 12 License months, it is available under a Creative Commons License (Attribution-NonCommercial 4.0 International), as described at http://creativecommons.org/licenses/by-nc/4.0/.

Email Alerting Receive free email alerts when new articles cite this article - sign up in the box at the Service top right corner of the article or click here. 